\title{
Efficacy and Safety of Adjusted-Dose Prasugrel Compared With Clopidogrel in Japanese Patients With Acute Coronary Syndrome - The PRASFIT-ACS Study -
}

Shigeru Saito, MD; Takaaki Isshiki, MD; Takeshi Kimura, MD; Hisao Ogawa, MD; Hiroyoshi Yokoi, MD; Shinsuke Nanto, MD; Morimasa Takayama, MD;

Kazuo Kitagawa, MD; Masakatsu Nishikawa, MD;

Shunichi Miyazaki, MD; Masato Nakamura, MD

\begin{abstract}
Background: Prasugrel is an antiplatelet agent that shows more prompt, potent, and consistent platelet inhibition than clopidogrel. The objective of this study was to confirm the efficacy and safety of prasugrel at loading/maintenance doses of $20 / 3.75 \mathrm{mg}$.
\end{abstract}

\begin{abstract}
Methods and Results: Japanese patients $(n=1,363)$ with acute coronary syndrome undergoing percutaneous coronary intervention were randomized to either prasugrel $(20 / 3.75 \mathrm{mg})$ or clopidogrel $(300 / 75 \mathrm{mg})$, both in combination with aspirin (81-330 mg for the first dose and 81-100 mg/day thereafter), for 24-48 weeks. The primary efficacy endpoint was the incidence of major adverse cardiovascular events (MACE) at 24 weeks, defined as a composite of cardiovascular death, nonfatal myocardial infarction, and nonfatal ischemic stroke. We compared the incidence of MACE between the 2 groups using point estimates. Safety outcomes included the incidence of bleeding events until 2 weeks after the last dose. The incidence of MACE at 24 weeks was $9.4 \%$ in the prasugrel group and $11.8 \%$ in the clopidogrel group (risk reduction $23 \%$, hazard ratio $0.77,95 \%$ confidence interval $0.56-1.07$ ). The incidence of non-coronary artery bypass graft-related major bleeding was similar in both groups (1.9\% vs. $2.2 \%$ ).
\end{abstract}

Conclusions: Prasugrel 20/3.75 mg was associated with a low incidence of ischemic events, similar to the results of TRITON-TIMI 38, and with a low risk of clinically serious bleeding in Japanese ACS patients. (Circ J 2014; 78: 1684-1692)

Key Words: Acute coronary syndrome; Clopidogrel; Major adverse cardiovascular events; Percutaneous coronary intervention; Prasugrel

$\mathbf{T}$ he incidence of coronary artery disease is steadily increasing in Japan, and is accompanied by an increase in the number of percutaneous coronary interventions (PCI). ${ }^{1}$ Patients undergoing PCI are usually started on dual antiplatelet therapy, consisting of aspirin and a thienopyridine, to prevent recurrent ischemia and stent thrombosis., ${ }^{2,3} \mathrm{Al}-$ though clopidogrel is often used with aspirin, the responses to clopidogrel vary in Asian patients. ${ }^{4}$

Received December 4, 2013; revised manuscript received March 6, 2014; accepted March 10, 2014; released online April 22, 2014 Time for primary review: 19 days

Division of Cardiology, Shonan Kamakura General Hospital, Kamakura (S.S.); Division of Cardiology, Teikyo University Hospital, Tokyo (T.I.); Graduate School of Medicine, Kyoto University, Kyoto (T.K.); Department of Cardiovascular Medicine, Graduate School of Medical Sciences, Kumamoto University, Kumamoto, and National Cerebral and Cardiovascular Center, Suita (H.O.); Cardiovascular Medicine Center, Fukuoka Sanno Hospital, Fukuoka, and International University of Health and Welfare, Ohtawara (H.Y.); Department of Advanced Cardiovascular Therapeutics, Graduate School of Medicine, Osaka University, Suita (S.N.); Sakakibara Heart Institute, Tokyo (M.T.); Department of Neurology, Graduate School of Medicine, Osaka University, Suita (K.K.); Institute of Human Research Promotion and Drug Development, Faculty of Medicine, Mie University, Tsu (M. Nishikawa); Division of Cardiology, Department of Medicine, Faculty of Medicine, Kinki University, Osakasayama (S.M.); and Division of Cardiovascular Medicine, Ohashi Medical Center, Toho University, Tokyo (M. Nakamura), Japan

Trial registration number: JapicCTI-101339

Financial Support: This study was sponsored by Daiichi Sankyo Co, Ltd (Tokyo, Japan).

Mailing address: Shigeru Saito, MD, Shonan Kamakura General Hospital, 1370-1 Okamoto, Kamakura 247-0072, Japan. E-mail: saito@ shonankamakura.or.jp

ISSN-1346-9843 doi:10.1253/circj.CJ-13-1482

All rights are reserved to the Japanese Circulation Society. For permissions, please e-mail: cj@j-circ.or.jp 


\section{Editorial p1577}

Prasugrel is a new-generation thienopyridine antiplatelet agent that provides more prompt, potent and consistent platelet inhibition than clopidogrel. ${ }^{5}$ The Trial to Assess Improvement in Therapeutic Outcomes by Optimizing Platelet Inhibition with Prasugrel Thrombolysis in Myocardial Infarction (TRITON-TIMI 38) in patients with acute coronary syndrome (ACS) undergoing PCI demonstrated that prasugrel resulted in significantly fewer ischemic events but had a higher incidence of bleeding compared with clopidogrel. ${ }^{6}$ Based on analyses of prespecified clinical features in TRITON-TIMI 38, age $\geq 75$ years, body weight $<60 \mathrm{~kg}$, and history of stroke or transient ischemic attack (TIA) were identified as risk factors for bleeding.

The Japanese package insert for clopidogrel includes precautions for careful administration in patients with a low body weight and in elderly patients because the risk of bleeding might be increased. Recent reports have also noted that other antithrombotic agents increased the bleeding risk in Japanese patients. ${ }^{7}$ Considering the higher average age and lower body weight of Japanese patients compared with Western patients, we considered that lower doses of prasugrel could diminish the risk of bleeding during dual antiplatelet therapy in Japanese patients. A recent Japanese Phase II trial (unpublished data) showed that administration of prasugrel (loading dose (LD)/ maintenance dose (MD): 20/3.75 mg for 12 weeks) was associated with a similar incidence of bleeding events compared with a standard clopidogrel regimen (LD/MD: 300/75 mg), independent of the age and body weight of patients.

The objective of the PRASFIT-ACS (PRASugrel compared with clopidogrel For Japanese patIen $\underline{T}$ s with $\underline{A C S}$ undergoing PCI) study was to confirm the effects of a prasugrel dosing regimen adjusted for Japanese patients (LD/MD: 20/3.75mg) on the incidence of major adverse cardiovascular events (MACE) and bleeding events, compared with the standard clopidogrel dosing regimen approved in Japan (LD/MD: 300/75 mg) as a control group, in ACS patients undergoing PCI.

\section{Methods}

\section{Study Design}

This randomized, double-blind, double-dummy, parallel-group study was conducted at 162 centers in Japan between December 2010 and June 2012. The database was locked in September 2012. The study consisted of a 24-48-week treatment period and a 14-day follow-up period.

\section{Patients}

The present study was conducted in Japanese ACS patients who satisfied all of the following criteria and were scheduled for coronary artery stenting: males/females aged $\geq 20$ years; presence of chest discomfort or ischemic symptoms lasting $\geq 10$ min within $72 \mathrm{~h}$ before randomization; ST-segment deviation $\geq 1 \mathrm{~mm}$, or T-wave inversion $\geq 3 \mathrm{~mm}$, or elevated levels of cardiac biomarkers for necrosis.

The major exclusion criteria were: refractory life-threatening ventricular arrhythmia; history or a current diagnosis of thrombocytopenia purpura or agranulocytosis; pancytopenia or aplastic anemia; history of intracranial bleeding; history of ischemic stroke/TIA; history of or predisposition to hemorrhagic disease; poorly controlled hypertension; severe hepatic or renal impairment; New York Heart Association (NYHA) grade IV heart failure; and administration of a thienopyridine drug within 5 days before starting the study drug or a thrombolytic drug within $24 \mathrm{~h}$ before starting the study drug. All of the patients provided informed consent before enrollment.

The present study was conducted in accordance with the Declaration of Helsinki and Good Clinical Practice, ${ }^{8,9}$ and was approved by the institutional review boards at all 162 participating centers.

\section{Treatments}

Eligible patients were randomized to either prasugrel or clopidogrel in a 1:1 ratio after they provided written informed consent. Randomization was performed using a uniform random numbers list generated using SAS software (SAS Institute, Cary, NC, USA).

The LD of prasugrel $(20 \mathrm{mg}$ ) or clopidogrel $(300 \mathrm{mg}$ ) was to be administered before PCI, except in urgent cases, when it could be administered up to $1 \mathrm{~h}$ after leaving the cardiac catheter laboratory. In most cases, PCI was performed immediately after obtaining informed consent, or at most several days after obtaining informed consent. The MD of prasugrel $(3.75 \mathrm{mg})$ or clopidogrel $(75 \mathrm{mg})$ was administered once daily after breakfast, starting on the day after the LD, and was continued for the remainder of the treatment period. Aspirin (81-330 mg for the first dose and $81-100 \mathrm{mg} /$ day thereafter) was concomitantly administered during the treatment period. The use of other antiplatelet drugs, anticoagulant drugs, thrombolytic drugs, or continuous administration of oral acidic non-steroidal antiinflammatory drugs was prohibited.

Clinic visits were scheduled every 2-4 weeks for the first 12 weeks, and every 12 weeks thereafter. Although 48 weeks was the recommended duration of drug administration, the investigators could complete drug administration at week 24 if deemed appropriate for the individual patient, taking into account stent type and the recommended duration of thienopyridine administration, as stated in the package inserts for the stents.

\section{Efficacy}

Patients who were administered at least one dose of the study drugs were followed up for 24-48 weeks to evaluate efficacy events. The primary efficacy endpoint was the incidence of MACE at 24 weeks, which was defined as a composite of cardiovascular death, nonfatal myocardial infarction (MI), and nonfatal ischemic stroke.

Cardiovascular death was defined as any death attributable to a cardiovascular event. Stroke was defined as the presence of neurological symptoms or signs consistent with stroke and confirmation by magnetic resonance imaging. Stroke was classified as ischemic (cerebral infarction) or non-ischemic stroke. Nonfatal MI was defined as events fulfilling at least one of the following 3 criteria. (1) In patients with normal creatine kinase (CK)-MB before PCI/coronary artery bypass grafting (CABG), $\mathrm{CK}-\mathrm{MB}$ had to be (a) $\geq 3$-fold the upper limit of normal (ULN) in 2 samples obtained, or $\geq 5$-fold the ULN in 1 sample obtained $<48 \mathrm{~h}$ after PCI, or (b) $\geq 10$-fold the ULN in 1 sample obtained $<48 \mathrm{~h}$ after CABG. Patients whose CK-MB exceeded the ULN before PCI/CABG had to show a transient decrease with a subsequent increase of $\geq 1$.5-fold the previous value and satisfy (a) or (b). (2) More than $48 \mathrm{~h}$ after PCI, the CK-MB or troponin levels had to be $\geq 2$-fold the ULN, accompanied by 1 or more of the following: new or recurrent sustained ischemic chest pain, hemodynamic decompensation, or new or recurrent ST elevation/depression $\geq 0.1 \mathrm{mV}$. (3) Abnormal Q waves had to persist for $\geq 0.04 \mathrm{~s}$.

As a secondary endpoint, we analyzed the incidence of the primary endpoint from randomization to completion of the 


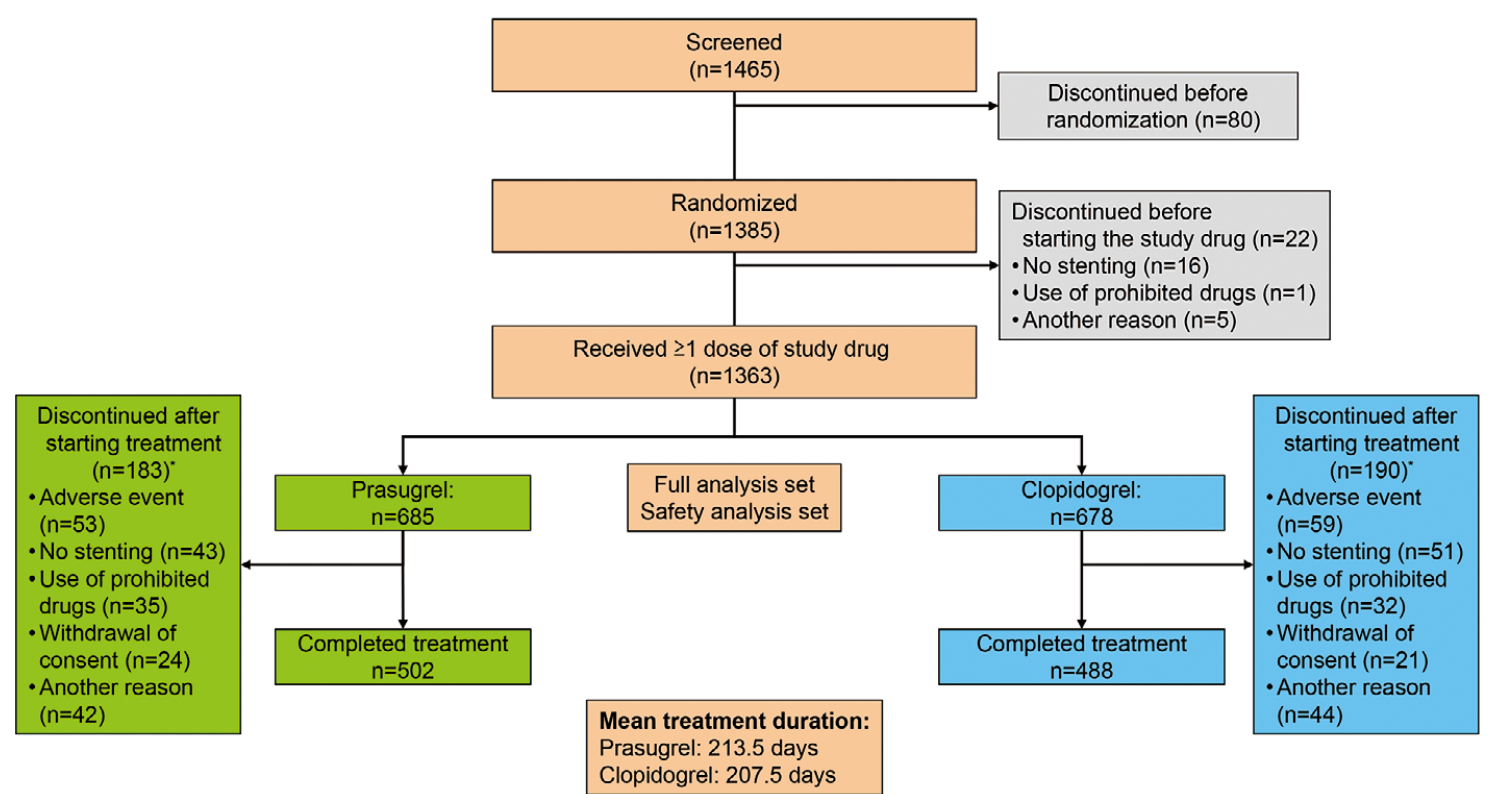

Figure 1. Patient disposition in the PRASFIT-ACS study (PRASugrel compared with clopidogrel For Japanese patlenTs with ACS undergoing $\mathrm{PCI}$ ). *Multiple reasons allowed.

follow-up period.

The other efficacy endpoints were the incidence of all-cause death, myocardial ischemia requiring rehospitalization, revascularization, and stent thrombosis (defined as definite or probable according to the Academic Research Consortium). ${ }^{10}$

\section{Safety}

The safety endpoint was the incidence of non-CABG-related bleeding events that occurred up until 2 weeks after the last dose. Bleeding events were classified as follows. (1) NonCABG-related TIMI major bleeding (major bleeding): intracranial bleeding or clinically significant bleeding accompanied by a decrease in hemoglobin $\geq 5 \mathrm{~g} / \mathrm{dl}$. (2) Non-CABG-related TIMI minor bleeding (minor bleeding): clinically significant bleeding accompanied by a decrease in hemoglobin of $3-<5 \mathrm{~g} / \mathrm{dl}$. (3) Clinically relevant non-major or minor bleeding: bleeding from critical sites (including retroperitoneal, intrapericardial, intravitreous/retinal, intraspinal, and intra-articular hemorrhage); gastrointestinal bleeding accompanied by decreased hemoglobin; gross hematuria not attributed to external factors; epistaxis requiring otolaryngology; gingival bleeding requiring dental treatment; bleeding requiring discontinuation of the study treatment at the investigator's discretion. These bleeding events were accompanied by a decrease in hemoglobin $<3 \mathrm{~g} / \mathrm{dl}$. (4) Other bleeding: all bleeding other than major bleeding, minor bleeding, or clinically relevant non-major or minor bleeding events.

Life-threatening bleeding was defined as a composite of the following major bleeding events: fatal bleeding, bleeding requiring intravenous inotropic medication, and bleeding requiring transfusion of $\geq 4$ units of red blood cells.

We also examined the incidence of treatment-emergency adverse events (AEs) that occurred up to 14-35 days after the last dose.

\section{Statistical Analysis}

All efficacy events were analyzed by the Efficacy Evaluation
Committee (comprising S.N., M.T. and K.K.) and bleeding events were analyzed by the Safety Evaluation Committee (comprising M. Nishikawa and S.M.); both committees were independent of the study sponsor. For events that were considered to satisfy the criteria of efficacy or bleeding events, the relevant committee re-evaluated and classified the events in a blinded manner. At the time of planning this study, we determined that it was not possible to enroll a sufficient number of Japanese patients to detect non-inferiority or superiority for the incidence of MACE between prasugrel and clopidogrel. However, prasugrel achieved a significant reduction in the incidence of MACE compared with clopidogrel in ACS patients in TRITON-TIMI 38. For these reasons, we determined whether the incidence of MACE was lower in the prasugrel group than in the clopidogrel group by determining point estimates, and ensuring that the hazard ratio (HR) was $<1.0$. Assuming that the incidence of MACE was $15 \%$ lower in the prasugrel group than in the clopidogrel group, ${ }^{6} 600$ patients were required per group. This would provide an estimated HR $<1.0$ at a probability $>0.8$.

Efficacy analyses were performed using the full analysis set, which consisted of all patients who took at least one dose of study drugs, did not have a serious violation of the study protocol, and who had data collected after starting administration of the study drug. Safety analyses were done in all patients who received at least one dose of the study drug and who did not have a serious violation of the study protocol.

HRs for the prasugrel group relative to the clopidogrel group, with 2 -sided $95 \%$ confidence intervals $(\mathrm{CI})$, were calculated for the primary efficacy endpoint and for other endpoints using the Cox proportional hazard model with adjustment for age ( $<75 \mathrm{vs.} \geq 75$ years) and number of lesions treated during PCI (no PCI, 1 lesion, multiple lesions). The incidence of events in each group was estimated using the Kaplan-Meier method. All analyses were performed used SAS version 9.2 (SAS Institute). 


\begin{tabular}{|c|c|c|c|}
\hline & Prasugrel $(n=685)$ & Clopidogrel $(n=678)$ & All patients $(n=1,363)$ \\
\hline & $n(\%)$ & $\mathrm{n}(\%)$ & $\mathrm{n}(\%)$ \\
\hline Female & $149(21.8)$ & $140(20.6)$ & $289(21.2)$ \\
\hline \multicolumn{4}{|l|}{ Age, years } \\
\hline Mean \pm SD & $65.4 \pm 11.4$ & $65.1 \pm 11.3$ & $65.2 \pm 11.4$ \\
\hline Median (range) & 65 (32-95) & $66(32-93)$ & $66(32-95)$ \\
\hline$\geq 75$ years & $165(24.1)$ & $148(21.8)$ & $313(23.0)$ \\
\hline Body weight, kg & $64.2 \pm 12.3$ & $64.4 \pm 11.2$ & $64.3 \pm 11.8$ \\
\hline$\leq 50$ & $85(12.4)$ & $72(10.6)$ & $157(11.5)$ \\
\hline$\leq 60$ & $266(38.9)$ & $240(35.4)$ & $506(37.2)$ \\
\hline Body mass index & $24.2 \pm 3.6$ & $24.2 \pm 3.3$ & $24.2 \pm 3.4$ \\
\hline Current smoker & $273(39.9)$ & 279 (41.2) & $552(40.5)$ \\
\hline \multicolumn{4}{|l|}{ ACS type } \\
\hline UA/NSTEMI & $343(50.1)$ & $337(49.7)$ & $680(49.9)$ \\
\hline UA & $156(22.8)$ & $124(18.3)$ & $280(20.5)$ \\
\hline NSTEMI & $187(27.3)$ & $213(31.4)$ & $400(29.3)$ \\
\hline STEMI & $340(49.6)$ & $341(50.3)$ & $681(50.0)$ \\
\hline \multicolumn{4}{|c|}{ No. of treated lesion sites (first $\mathrm{PCl}$ ) } \\
\hline 1 & $470(68.6)$ & $455(67.1)$ & $925(67.9)$ \\
\hline$>1$ & $181(26.4)$ & $182(26.8)$ & $363(26.6)$ \\
\hline No first $\mathrm{PCl}$ & $34(5.0)$ & $41(6.0)$ & $75(5.5)$ \\
\hline Hypertension & 495 (72.3) & $491(72.4)$ & 986 (72.3) \\
\hline Dyslipidemia & $516(75.3)$ & $500(73.7)$ & $1,016(74.5)$ \\
\hline Diabetes mellitus & $250(36.5)$ & $237(35.0)$ & $487(35.7)$ \\
\hline Prior MI & $34(5.0)$ & $35(5.2)$ & $69(5.1)$ \\
\hline Prior revascularizations & $40(5.8)$ & $45(6.6)$ & $85(6.2)$ \\
\hline Prior CABG & $6(0.9)$ & $4(0.6)$ & $10(0.7)$ \\
\hline Prior TLR & $15(2.2)$ & $13(1.9)$ & $28(2.1)$ \\
\hline $\mathrm{CrCl}(\mathrm{ml} / \mathrm{min})$ & $n=622$ & $n=625$ & $n=1,247$ \\
\hline$>60$ & $486(78.1)$ & $507(81.1)$ & $993(79.6)$ \\
\hline$\leq 60$ & $136(21.9)$ & $118(18.9)$ & $254(20.4)$ \\
\hline \multicolumn{4}{|l|}{ Concomitant therapy } \\
\hline Proton-pump inhibitor & $282(41.2)$ & $287(42.3)$ & $569(41.7)$ \\
\hline Statin & $346(50.5)$ & $328(48.4)$ & $674(49.4)$ \\
\hline Ca-blocker & $184(26.9)$ & $159(23.5)$ & $343(25.2)$ \\
\hline$\beta$-blocker & $122(17.8)$ & $114(16.8)$ & 236 (17.3) \\
\hline \multicolumn{4}{|l|}{ Stent type } \\
\hline Bare metal & $353(51.5)$ & $353(52.1)$ & $706(51.8)$ \\
\hline Drug-eluting & 291 (42.5) & $278(41.0)$ & 569 (41.7) \\
\hline
\end{tabular}

Values are $\mathrm{n}(\%)$ or mean \pm standard deviation.

ACS, acute coronary syndrome; $\mathrm{CABG}$, coronary artery bypass grafting; $\mathrm{CrCl}$, creatinine clearance (estimated using the Cockcroft-Gault formula); MI, myocardial infarction; NSTEMI, non-ST-segment elevation myocardial infarction; $\mathrm{PCl}$, percutaneous coronary intervention; STEMI, ST-segment elevation myocardial infarction; TLR, target lesion revascularization; UA, unstable angina.

\section{Results}

\section{Study Population}

Of 1,465 patients who gave informed consent, 1,385 were randomized and 1,363 received either prasugrel $(n=685)$ or clopidogrel $(n=678)$ (Figure 1$) ; 80$ patients discontinued before randomization and 22 patients discontinued after randomization but before administration of the LD because of the absence of significant stenosis, unsuitability for stent implantation, or failure to meet eligibility criteria. Overall, 183 and 190 patients in the prasugrel and clopidogrel groups, respectively, discontinued after starting the study drug.

Table 1 shows the characteristics of the 1,363 patients included in the full analysis set. There were no differences be- tween the 2 groups in terms of baseline demographic factors, medical history, concomitant therapy, and procedural variables. The mean duration of study drug administration was 213.5 days in the prasugrel group and 207.5 days in the clopidogrel group. The median dose of aspirin at the time of LD was $200 \mathrm{mg}$, and that during follow-up was $100 \mathrm{mg}$ in both treatment groups. The mean duration of aspirin administration was 230.7 days in the prasugrel group and 224.0 days in the clopidogrel group.

\section{Efficacy}

Table 2 compares the efficacy endpoints between the prasugrel and clopidogrel groups. The primary efficacy endpoint, the incidence of MACE at 24 weeks, was 9.4\% (95\% CI 7.3-11.8) 


\begin{tabular}{|c|c|c|c|}
\hline \multirow{2}{*}{ Efficacy endpoint } & Prasugrel $(n=685)$ & Clopidogrel $(n=678)$ & \multirow{2}{*}{ HR (95\% Cl) } \\
\hline & $n(\%)^{\star}$ & $\mathbf{n}(\%)^{\star}$ & \\
\hline MACE through to week 24 & $64(9.4)$ & $80(11.8)$ & $0.77(0.56-1.07)$ \\
\hline CV death & $9(1.3)$ & $6(0.9)$ & $1.45(0.51-4.07)$ \\
\hline Nonfatal MI & $52(7.6)$ & $68(10.1)$ & $0.74(0.52-1.06)$ \\
\hline Nonfatal ischemic stroke & $3(0.4)$ & $7(1.0)$ & $0.43(0.11-1.68)$ \\
\hline \multicolumn{4}{|c|}{ Components of MACE through to week 24} \\
\hline All-cause death & $9(1.3)$ & $8(1.2)$ & $1.09(0.42-2.83)$ \\
\hline Nonfatal stroke & $3(0.4)$ & $8(1.2)$ & $0.38(0.10-1.43)$ \\
\hline Revascularization & $31(4.6)$ & $32(4.8)$ & $0.96(0.58-1.57)$ \\
\hline Stent thrombosis & $3(0.4)$ & $5(0.7)$ & $0.60(0.14-2.51)$ \\
\hline MACE through to week 48 & $74(11.1)$ & $84(12.7)$ & $0.85(0.62-1.16)$ \\
\hline CV death & $10(1.5)$ & $8(1.4)$ & $1.21(0.48-3.06)$ \\
\hline Nonfatal MI & $58(8.7)$ & $68(10.1)$ & $0.83(0.58-1.17)$ \\
\hline Nonfatal ischemic stroke & $6(1.0)$ & $9(1.4)$ & $0.66(0.24-1.86)$ \\
\hline \multicolumn{4}{|c|}{ Components of MACE through to week 48} \\
\hline All-cause death & $11(1.8)$ & $11(2.1)$ & $0.96(0.42-2.22)$ \\
\hline Nonfatal stroke & $6(1.0)$ & $10(1.5)$ & $0.60(0.22-1.64)$ \\
\hline Revascularization & $107(20.0)$ & $96(19.4)$ & $1.10(0.84-1.45)$ \\
\hline Stent thrombosis & $4(0.6)$ & $5(0.7)$ & $0.80(0.21-2.96)$ \\
\hline MACE $^{\ddagger}$ while on treatment ${ }^{\dagger}$ & $67(10.7)$ & $78(12.0)$ & $0.83(0.60-1.15)$ \\
\hline CV death & $4(0.6)$ & $5(0.8)$ & $0.76(0.20-2.84)$ \\
\hline Nonfatal MI & $58(9.3)$ & $67(10.2)$ & $0.84(0.59-1.19)$ \\
\hline Nonfatal ischemic stroke & $5(0.9)$ & $6(1.1)$ & $0.82(0.25-2.70)$ \\
\hline \multicolumn{4}{|c|}{ Components of MACE while on treatment ${ }^{\dagger}$} \\
\hline All-cause death & $5(1.1)$ & $5(0.8)$ & $0.95(0.27-3.28)$ \\
\hline Nonfatal stroke & $5(0.9)$ & $7(1.2)$ & $0.71(0.22-2.23)$ \\
\hline Revascularization & $83(19.5)$ & $72(17.2)$ & $1.13(0.83-1.55)$ \\
\hline Stent thrombosis & $4(0.6)$ & $5(0.8)$ & $0.79(0.21-2.95)$ \\
\hline
\end{tabular}

${ }^{*}$ Cumulative incidence. ${ }^{+}$From first dose of study treatment until 14 days after last treatment. ${ }^{\circ}$ Composite of cardiovascular death, nonfatal $\mathrm{Ml}$, and nonfatal ischemic stroke.

$\mathrm{Cl}$, confidence interval; CV, cardiovascular; HR, hazard ratio; MACE, major adverse cardiac events. Other abbreviations as in Table 1.

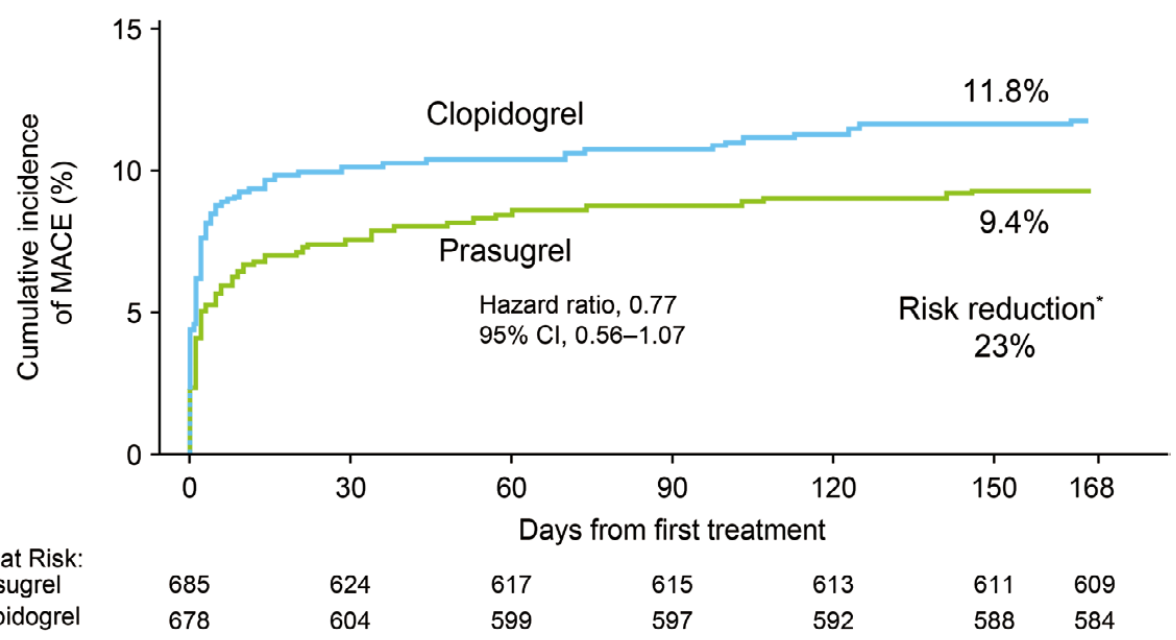

Figure 2. Kaplan-Meier estimates of the incidence of the primary efficacy endpoint, major coronary adverse events (MACE: composite of cardiovascular death, nonfatal myocardial infarction, and nonfatal ischemic stroke) at 24 weeks in the PRASFIT-ACS study (PRASugrel compared with clopidogrel For Japanese patlenTs with ACS undergoing PCI). Based on full analysis set. *Risk reduction was calculated as: (1-hazard ratio) $\times 100$. 


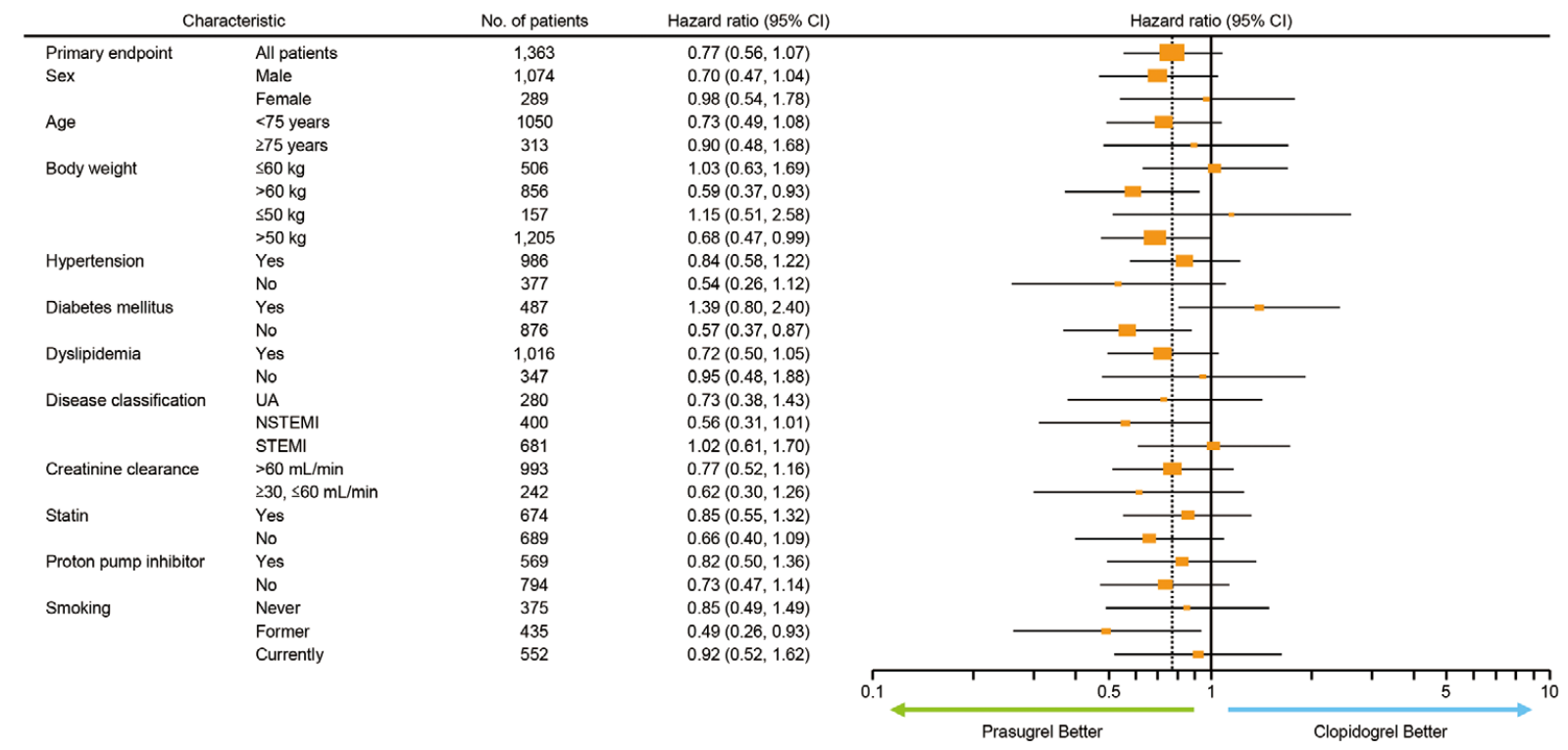

Figure 3. Hazard ratios (HRs) and risk reduction for the primary efficacy endpoint and other efficacy events in prespecified subgroups of patients from start of drug administration to 24 weeks in the PRASFIT-ACS study (PRASugrel compared with clopidogrel For Japanese patlenTs with ACS undergoing PCI). Based on the full analysis set. The HRs are based on Cox regression using age ( $\geq 75,<75$ years) and the number of treated lesion sites (first PCl) as covariates. If a characteristic was used as a covariate, it was removed from the Cox regression model. UA, unstable angina; NSTEMI, non-ST-segment elevation myocardial infarction; STEMI, ST-segment elevation myocardial infarction; $\mathrm{CrCl}$, creatinine clearance (estimated using the Cockcroft-Gault formula).

\begin{tabular}{|c|c|c|c|c|c|c|}
\hline \multirow{3}{*}{ Bleeding event } & \multicolumn{3}{|c|}{ All } & \multicolumn{3}{|c|}{ Spontaneous } \\
\hline & $\begin{array}{c}\text { Prasugrel } \\
(n=685)\end{array}$ & $\begin{array}{c}\text { Clopidogrel } \\
(n=678)\end{array}$ & HR $(95 \% \mathrm{Cl})$ & $\begin{array}{c}\text { Prasugrel } \\
(n=685)\end{array}$ & $\begin{array}{c}\text { Clopidogrel } \\
(n=678)\end{array}$ & $\mathrm{HR}(95 \% \mathrm{Cl})$ \\
\hline & n (\%) $)^{\star}$ & n (\%) $)^{\star}$ & & n (\%)* & n (\%)* & \\
\hline Overall bleeding events & $341(49.8)$ & $247(36.4)$ & $1.48(1.25-1.74)$ & $115(16.8)$ & $103(15.2)$ & $1.09(0.83-1.42)$ \\
\hline Major TIMI bleeding & $13(1.9)$ & $15(2.2)$ & $0.82(0.39-1.73)$ & $4(0.6)$ & $6(0.9)$ & $0.64(0.18-2.27)$ \\
\hline Life-threatening bleeding & $4(0.6)$ & $7(1.0)$ & $0.54(0.16-1.85)$ & $1(0.1)$ & $4(0.6)$ & $0.24(0.03-2.16)$ \\
\hline Fatal bleeding & $2(0.3)$ & $1(0.1)$ & $1.77(0.16-19.54)$ & $1(0.1)$ & $1(0.1)$ & $0.90(0.06-14.45)$ \\
\hline Minor TIMI bleeding & $27(3.9)$ & $15(2.2)$ & $1.76(0.94-3.31)$ & $7(1.0)$ & $6(0.9)$ & $1.10(0.37-3.27)$ \\
\hline Clinically relevant bleeding & $29(4.2)$ & $39(5.8)$ & $0.72(0.44-1.16)$ & $24(3.5)$ & $35(5.2)$ & $0.66(0.39-1.11)$ \\
\hline Other bleeding & $298(43.5)$ & $209(30.8)$ & $1.51(1.26-1.80)$ & $85(12.4)$ & $67(9.9)$ & $1.24(0.90-1.71)$ \\
\hline Major or minor TIMI bleeding & $39(5.7)$ & $29(4.3)$ & $1.30(0.81-2.11)$ & $11(1.6)$ & $12(1.8)$ & $0.87(0.38-1.97)$ \\
\hline Major, minor, or clinically relevant bleeding & $66(9.6)$ & $65(9.6)$ & $0.98(0.70-1.38)$ & $34(5.0)$ & $45(6.6)$ & $0.72(0.46-1.13)$ \\
\hline Bleeding events leading to discontinuation & $16(2.3)$ & $20(2.9)$ & $0.76(0.40-1.48)$ & $9(1.3)$ & $12(1.8)$ & $0.72(0.30-1.71)$ \\
\hline
\end{tabular}

${ }^{*}$ Incidence $(\%)=\mathrm{n}$ for each bleeding event $/ \mathrm{n}$ for each group (prasugrel or clopidogrel) $\times 100 \%$. TIMI, thrombolysis in myocardial infarction. Other abbreviations as in Table 2.

in the prasugrel group and $11.8 \%$ (95\% CI 9.5-14.5) in the clopidogrel group. The HR was 0.77 (95\% CI 0.56-1.07), corresponding to a risk reduction (1-HR) of $23 \%$ for prasugrel (Figures 2,3).

During the first 24 weeks of the study, the incidence of nonfatal MI, the most frequent MACE, was $7.6 \%$ in the prasugrel group and $10.1 \%$ in the clopidogrel group. The HR was 0.74 (95\% CI 0.52-1.06). The incidence of cardiovascular death was $1.3 \%$ in the prasugrel group and $0.9 \%$ in the clopidogrel group, and the incidence of nonfatal stroke was $0.4 \%$ in the prasugrel group and $1.0 \%$ in the clopidogrel group. The incidence of stent thrombosis $(0.4 \%$ vs. $0.7 \%)$ and of revascular- ization ( $4.6 \%$ vs. $4.8 \%)$ was similar in the prasugrel and clopidogrel groups. In the subgroup analysis of MACE at 24 weeks, there were no subgroups with significantly worse outcomes in the prasugrel group than in the clopidogrel group (Table 2, Figure 3). The incidence of stent thrombosis at 24 weeks was similar in both groups, irrespective of whether the patient received a bare metal stent $(0.6 \%$ vs. $0.8 \%)$ or a drug-eluting stent $(0.3 \%$ vs. $0.7 \%)$. The type/brand of stent did not influence the incidence of MACE in either group (data not shown).

There were slight increases in the overall incidence of MACE and of each component between 24 and 48 weeks. The HRs for MACE and its components at 48 weeks were similar 


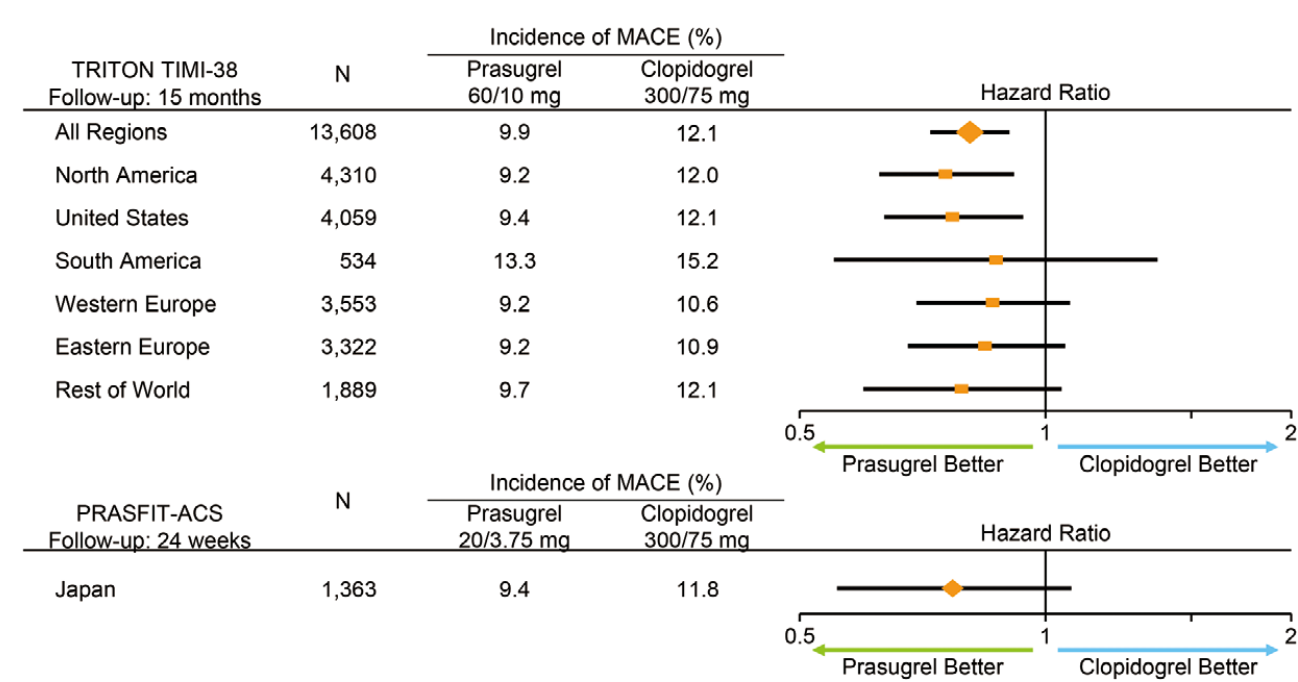

Figure 4. Rates of primary efficacy endpoints in the present (PRASFIT-ACS) study and in TRITON-TIMI 38. The incidence of the primary efficacy endpoint (major adverse cardiac events [MACE]) and the hazard ratios were similar between the 2 studies. Modified from Ruff et al. ${ }^{12}$

to those at 24 weeks (Table 2).

The incidence of MACE from the first dose until 14 days after the last dose was similar to that at 24 weeks (Table 2). The incidence of cardiovascular death up to 14 days after the last dose was $0.6 \%$ in the prasugrel group and $0.8 \%$ in the clopidogrel group. Post hoc analyses of the efficacy endpoints through to week 24 and until 14 days after the last dose confirmed that the incidence of cardiovascular death was similar between the 2 groups (data not shown).

\section{Bleeding Events}

Table 3 shows the incidence of non-CABG-related bleeding events that occurred up until 14 days after the last dose in both groups. Major bleeding events, which were the key safety endpoint in TRITON-TIMI 38, occurred in $1.9 \%(13 / 685)$ of patients treated with prasugrel and in $2.2 \%(15 / 678)$ of patients treated with clopidogrel. The incidences of major spontaneous bleeding and life-threatening bleeding events were similar in both groups. Only 2 patients treated with prasugrel and 1 patient treated with clopidogrel had a fatal major bleeding event. We also examined the incidence of bleeding events in subgroups of patients with a high risk of bleeding, as reported in TRITON-TIMI $38,{ }^{6}$ namely those aged $\geq 75$ years or weighing $\leq 60 \mathrm{~kg}$. In the present study, the incidence of major bleeding among patients aged $\geq 75$ years $(3.6 \%[6 / 165]$ vs. $4.1 \%$ [6/148]) or those weighing $\leq 60 \mathrm{~kg}(3.4 \%$ [9/266] vs. $3.8 \%$ [9/240]) was comparable between the prasugrel and clopidogrel groups. We also examined the incidence of major bleeding in the subgroup of patients weighing $\leq 50 \mathrm{~kg}$, which was defined as low body weight in the Japanese Phase II study (unpublished data). The incidence of major bleeding was 5.9\% (5/85) in the prasugrel group and $2.8 \%(2 / 72)$ in the clopidogrel group, but the incidence of spontaneous major bleeding was very low in both groups $(2.4 \%$ [2/85] vs. $1.4 \%$ [1/72]).

The incidence of major or minor bleeding events, which was the key safety endpoint in the Japanese Phase II trial, was $5.7 \%(39 / 685)$ in the prasugrel group and $4.3 \%$ (29/678) in the clopidogrel group, but the incidence of spontaneous bleeding events with non-external causes was similar in both groups $(1.6 \%,[11 / 685]$ vs. $1.8 \%,[12 / 678])$. Regarding the incidences of major or minor bleeding events with external causes, complications of PCI occurred in $2.8 \%$ and $1.8 \%$ of patients in the prasugrel and clopidogrel groups, respectively, with other causes noted in $1.3 \%$ and $0.7 \%$ of patients in the prasugrel and clopidogrel groups, respectively.

The incidences of non-CABG-related major, minor, or clinically relevant bleeding, and bleeding events leading to discontinuation were similar in both groups.

AEs

Treatment-emergency AEs occurred in $89.8 \%$ (615/685) of patients treated with prasugrel and in $88.5 \%(600 / 678)$ of patients treated with clopidogrel (Table S1). Serious AEs occurred in $27.4 \%(188 / 685)$ and $25.4 \%(172 / 678)$ of patients treated with prasugrel and clopidogrel, respectively. AEs requiring drug withdrawal occurred in $7.9 \%$ (54/685) and 9.0\% $(61 / 678)$ of patients treated with prasugrel and clopidogrel, respectively. AEs related to the study drug that occurred in $\geq 5 \%$ of patients in both groups were bleeding-related events (Table S1).

\section{Discussion}

In this study, we confirmed that the HR for the prasugrel $20 / 3.75 \mathrm{mg}$ group relative to the clopidogrel $300 / 75 \mathrm{mg}$ group was $<1.0$ for the incidence of MACE (HR $0.77,95 \%$ CI $0.56-$ 1.07 ), corresponding to a risk reduction of $23 \%$ for prasugrel.

To show superiority of a drug relative to a comparator, it is usually necessary to enroll a large number of patients, especially if an active comparator is being used. Indeed, in TRITONTIMI 38, more than 13,000 patients were enrolled to compare the efficacy and safety of prasugrel and clopidogrel. For the present study, however, it was not possible to enroll a sufficient number of Japanese patients to achieve a sample size that would be able to detect statistical superiority. However, prasugrel achieved a significant reduction in the incidence of 
MACE compared with clopidogrel in the ACS patients in TRITON-TIMI 38. Although the present study and TRITONTIMI 38 differed in terms of the prasugrel dosing regimen and the follow-up period, both studies were fairly similar in terms of disease setting, inclusion criteria, and primary endpoint. Furthermore, the clopidogrel dosing regimen was identical and most MACE occurred before 24 weeks in TRITON-TIMI 38. The same trend was observed in a Japanese ACS registry. ${ }^{11}$ For these reasons, we determined whether the HR for prasugrel relative to clopidogrel was $<1.0$ for the incidence of MACE at 24 weeks by determining point estimates. As shown in Figure 4, the incidence of MACE in the PRASFIT-ACS study using the lower dose of prasugrel of $20 / 3.75 \mathrm{mg}$ was similar to that in TRITON-TIMI 38. ${ }^{12}$ The incidence of MACE at 24 weeks in both groups in the present study seemed to be slightly higher than in TRITON-TIMI 38. The present study included greater proportions of patients aged $\geq 75$ years and patients with multiple lesions treated with the first PCI compared with TRITON-TIMI 38. The incidence of MACE in each of these subgroups was higher in the results of blind review; thus, these differences between the 2 trials might explain the differences in the incidence of MACE. However, most MACE occurred in the first 24 weeks of TRITON-TIMI 38 , and the HR obtained in the PRASFIT-ACS study (HR $0.77,95 \%$ CI $0.56-1.07$ ) was similar to that obtained in the overall cohort of TRITON-TIMI 38 (HR $0.81,95 \%$ CI $0.73-$ $0.90)$. In addition, the HR in the overall cohort and the HR at 180 days were similar in TRITON-TIMI 38 (data not shown). Based on these results, we considered the efficacy of prasugrel in the PRASFIT-ACS study was similar to that shown in the TRITON-TIMI 38 study. This trend for the incidence of MACE in the prasugrel group was maintained throughout the 24 weeks of treatment and until the end of the 48 -week treatment period.

We determined the composite endpoint used in TRITONTIMI $38,{ }^{6}$ with the exception of nonfatal non-ischemic stroke. In TRITON-TIMI 38, MACE included nonfatal stroke irrespective of the presence/absence of ischemia. ${ }^{6}$ Based on the pharmacological effects of thienopyridines, we excluded nonfatal non-ischemic stroke from the primary efficacy endpoint. Because nonfatal non-ischemic stroke occurred in only 1 patient, who was treated with clopidogrel, exclusion of this component did not affect the primary efficacy outcome. Consequently, the incidence of the primary endpoint in our study was comparable with that in TRITON-TIMI 38.

The incidence of cardiovascular death during the first 24 weeks in our study was $1.3 \%$ in the prasugrel group and $0.9 \%$ in the clopidogrel group. However, the incidence of cardiovascular death until 14 days after the last dose was similar in both treatment groups. This difference in cardiovascular mortality was because of 6 deaths that occurred $>14$ days after switching from prasugrel to clopidogrel or from prasugrel to no drug treatment. Based on these results, we must be careful when interpreting the incidence of cardiovascular death in both treatment groups.

The adjusted dosing regimen for Japanese patients should minimize the risk of bleeding. The incidence of non-CABGrelated major bleeding and the incidence of major, minor, or clinically relevant bleeding was almost the same in the prasugrel and clopidogrel groups. In TRITON-TIMI 38, prasugrel resulted in a significantly higher incidence of major bleeding compared with clopidogrel, but this trend was not observed in the PRASFIT-ACS study, even though the incidence of major or minor bleeding events was $5.7 \%$ in the prasugrel group and $4.3 \%$ in the clopidogrel group. In addition, the incidence of spontaneous major or minor bleeding events was almost the same in both groups (1.6\% vs. $1.8 \%)$. In the subgroup analysis of patients aged $\geq 75$ years or weighing $\leq 60 \mathrm{~kg}$, which were identified as risk factors in TRITON-TIMI 38, the incidence of major bleeding was similar between the prasugrel and clopidogrel groups. Among patients weighing $\leq 50 \mathrm{~kg}$, which was defined as low body weight in the Japanese Phase II study, the incidence of major bleeding was $5.9 \%$ in the prasugrel group and $2.8 \%$ in the clopidogrel group, but the incidence of spontaneous major bleeding was very low in both treatment groups. Therefore, we consider that the adjusted dosing regimen for Japanese patients minimizes the risk of bleeding.

The incidence of treatment-emergency AEs was similar in both treatment groups. AEs related to the study drug occurred in just under half of the patients in both groups, but all of the events that occurred in $\geq 5 \%$ of patients in both groups were bleeding-related events (eg, subcutaneous bleeding and nosebleeds). In almost all cases, patients recovered with appropriate treatment.

As shown in Figure 3, none of the subgroups showed significantly worse outcomes in the prasugrel group than in the clopidogrel group. Therefore, we considered that the efficacy of prasugrel in the prespecified subgroups was consistent with that in the overall cohort of the PRASFIT-ACS study. However, unexpectedly, the direction of the HR was opposite in patients with and without diabetes mellitus. The HR was 1.39 (95\% CI 0.80-2.40) in patients with diabetes mellitus and 0.57 (95\% CI 0.37-0.87) in patients without diabetes mellitus. The incidence of MACE in patients in the clopidogrel group with and without diabetes mellitus was $9.7 \%$ and $12.9 \%$, respectively, which was quite different from previous reports. ${ }^{6,13,14}$ However, in the prasugrel group, the incidence of MACE in patients with diabetes mellitus was similar to that described in previous studies, including the TRITON-TIMI 38 study. ${ }^{6} \mathrm{Al}-$ though it is difficult to provide a clear explanation for these findings, we suspect the small sample size of the subgroups may have contributed.

We need antiplatelet drugs to reduce ischemic events. However, an increase in platelet inhibition may increase the risk of bleeding. In the PRASFIT-ACS study, the adjusted dosing regimen of prasugrel for Japanese patients provided similar efficacy to that reported in TRITON-TIMI 38, without an increased risk of bleeding compared with clopidogrel. We think that the main contributor to these outcomes of the PRASFITACS study was the use of an adjusted dosing regimen of prasugrel that balances the benefits and possible risks of prasugrel in Japanese patients.

\section{Study Limitations}

The main limitation of this study was the relatively small sample size. It was not feasible to conduct a clinical trial in Japan that could enroll a sufficient number of patients to detect statistical superiority or non-inferiority of prasugrel relative to clopidogrel for the incidence of MACE. This issue might explain why the $95 \%$ CIs for some comparisons (eg, MACE through to week 24 or 48 ) included 1 . However, the present study is the largest Japanese clinical trial to date of patients with ACS undergoing PCI, and the results are similar to those reported in TRITON-TIMI 38 based on prespecified point estimates of MACE for the prasugrel and clopidogrel groups.

Another limitation of the present study is that we excluded patients with a history of stroke and/or TIA. In TRITON-TIMI 38 , patients with these conditions did not benefit from prasugrel treatment because they tended to experience more major bleeding events; therefore, we considered that such patients should 
not be included for prasugrel treatment in the present study. Nevertheless, this factor should be taken into account when comparing the results of this study with those of TRITONTIMI 38.

\section{Conclusions}

A prasugrel dosing regimen (LD/MD: $20 / 3.75 \mathrm{mg}$ ) that was adjusted for Japanese patients with ACS undergoing PCI was associated with a low incidence of MACE, similar to the results of TRITON-TIMI 38, and with a low risk of clinically serious bleeding in Japanese ACS patients.

\section{Acknowledgments}

The authors greatly appreciate the contributions of all the investigators and other clinical/research staff involved in the present study. In particular, we acknowledge the contributions of Yasuo Ikeda, MD (Waseda University), who acted as an advisor during the evaluation of pharmacodynamics, and the following members of the Independent Data Monitoring Committee: Hideki Origasa, PhD (Toyama University), Kazuhiro Sase, MD (Juntendo University), Kazuo Umemura, MD (Hamamatsu University), and Takanari Kitazono, MD (Kyushu University). We also acknowledge the support of the institutions that participated in or helped implement the PRASFITACS study, as listed in Supplementary File 1.

\section{Disclosures}

The authors declare the following interests: Shigeru Saito is a medical advisor for Terumo and has received honoraria from Abbot Vascular Japan, Boston Scientific Japan, and Medtronic; Masato Nakamura has received honoraria from AstraZeneca K.K., Daiichi Sankyo Co, Ltd, and Sanofi K.K.; Takaaki Isshiki has received honoraria from AstraZeneca K.K., Daiichi Sankyo Co, Ltd, Otsuka Pharmaceutical Co Ltd, and Sanofi K.K. Takeshi Kimura has received honoraria, clinical research funding, and other research funding from Daiichi Sankyo Co, Ltd and Sanofi K.K.; Hisao Ogawa has received honoraria from AstraZeneca K.K., Bayer Yakuhin, Ltd, Boehringer Ingelheim Japan, Daiichi Sankyo Co, Ltd, Dainippon Sumitomo Pharma Co, Ltd, Eisai Co, Ltd, Kyowa Hakko Kirin Co, Ltd, Mitsubishi Tanabe Pharma Corporation, MSD K.K., Pfizer Japan Inc, Sanofi K.K., and Takeda Pharmaceutical Co, Ltd, clinical research funding from Daiichi Sankyo Co, Ltd, and other research funding from Astellas Pharma Inc, AstraZeneca K.K., Boehringer Ingelheim Japan, Chugai Pharmaceutical Co, Ltd, Daiichi Sankyo Co, Ltd, Eisai Co, Ltd, Kowa Company, Ltd, Mitsubishi Tanabe Pharma Corporation, MSD K.K., Novartis Pharma K.K., Otsuka Pharmaceutical Co, Ltd, Shionogi \& Co, Ltd, and Takeda Pharmaceutical Co, Ltd; Shinsuke Nanto has received honoraria from Daiichi Sankyo Co, Ltd, Medtronic, Otsuka Pharmaceutical Co, Ltd, Sanofi K.K., and Takeda Pharmaceutical Co, Ltd, clinical research funding from Abbott Vascular Japan and Terumo, other research funding from Abbott Vascular Japan, Boston Scientific Japan, Daiichi Sankyo Co, Medtronic, Ltd, Sanofi K.K., and St. Jude Medical Japan, and an endowment from Terumo; Morimasa Takayama is a clinical advisor for Abbott Vascular Japan and Kaneka Medics Co, Ltd, and has received honoraria from Daiichi Sankyo Co, Ltd; Kazuo Kitagawa has received honoraria from Sanofi K.K., and other research funding from Boehringer Ingelheim Japan and Sanofi K.K.; Masakatsu Nishikawa is a medical advisor for and holds stock in D. Western Therapeutics Inc, and has received honoraria from Daiichi Sankyo Co, Ltd and Otsuka Pharmaceutical Co, Ltd. and other research funding from Otsuka Pharmaceutical Co; Shunichi Miyazaki has received other research funding from MSD K.K.; Hiroyoshi Yokoi has no conflicts of interest to declare.

\section{References}

1. JJCRAC/Data Center. Report of survey on treatment for cardiovas- cular disease 2008 in Japanese. http://www.j-circ.or.jp/jittai_chosa/ jittai_chosa2008web.pdf (accessed July 16, 2010).

2. Anderson JL, Wright RS, Adams CD, Bridges CR, Casey DE Jr, Ettinger SM, et al. 2012 ACCF/AHA focused update of the guideline for the management of patients with unstable angina/non-ST-elevation myocardial infarction (updating the 2007 guideline and replacing the 2011 focused update): A report of the American College of Cardiology Foundation/American Heart Association Task Force on Practice Guidelines. J Am Coll Cardiol 2012; 60: 645-681.

3. Kushner FG, Hand M, Smith SC Jr, King SB 3rd, Anderson JL, Antman EM, et al. 2009 Focused updates: ACC/AHA Guidelines for the Management of Patients With ST-Elevation Myocardial Infarction (updating the 2004 Guideline and 2007 Focused Update) and ACC/AHA/ SCAI Guidelines on Percutaneous Coronary Intervention (updating the 2005 Guideline and 2007 Focused Update): A report of the American College of Cardiology Foundation/American Heart Association Task Force on Practice Guidelines. Circulation 2009; 120: 2271-2306.

4. Park KW, Kim HS. Options to overcome clopidogrel response variability. Circ J 2012; 76: 287-292.

5. Wiviott SD, Trenk D, Frelinger AL, O'Donoghue M, Braunwald E, Michelson AD, et al. Prasugrel compared with high loading- and maintenance-dose clopidogrel in patients with planned percutaneous coronary intervention: The Prasugrel in Comparison to Clopidogrel for Inhibition of Platelet Activation and Aggregation-Thrombolysis in Myocardial Infarction 44 trial. Circulation 2007; 116: 2923-2932.

6. Wiviott SD, Braunwald E, McCabe CH, Montalescot G, Ruzyllo W, Gottlieb S, et al. Prasugrel versus clopidogrel in patients with acute coronary syndromes. N Engl J Med 2007; 357: 2001 - 2015.

7. Hori M, Connolly SJ, Ezekowitz MD, Reilly PA, Yusuf S, Wallentin L. Efficacy and safety of dabigatran vs. warfarin in patients with atrial fibrillation: Sub-analysis in Japanese population in RE-LY trial. Circ J 2011; 75: 800-805.

8. Pharmaceutical and Food Safety Bureau, Ministry of Health, Labour and Welfare. Basic principle on global clinical trials. September 2007. Tokyo: The Bureau.

9. ICH Steering Committee. Ethnic factors in the acceptability of foreign clinical data (ICH-E5(R1)). March 1998.

10. Mauri L, Hsieh WH, Massaro JM, Ho KKL, D'Agostino R, Cutlip DE. Stent thrombosis in randomized clinical trials of drug-eluting stents. N Engl J Med 2007; 356: 1020-1029.

11. Daida H, Miyauchi K, Ogawa H, Yokoi H, Matsumoto M, Kitakaze $\mathrm{M}$, et al. Management and two-year long-term clinical outcome of acute coronary syndrome in Japan: Prevention of atherothrombotic incidents following ischemic coronary attack (PACIFIC) registry. Circ J 2013; 77: 934-943.

12. Ruff CT, Giugliano RP, Antman EM, Murphy SA, Lotan C, Heuer $\mathrm{H}$, et al. Safety and efficacy of prasugrel compared with clopidogrel in different regions of the world. Int J Cardiol 2012; 155: 424-429.

13. Ehara N, Morimoto T, Furukawa Y, Shizuta S, Taniguchi R, Nakagawa $\mathrm{Y}$, et al. Effect of baseline glycemic level on long-term cardiovascular outcomes after coronary revascularization therapy in patients with type 2 diabetes mellitus treated with hypoglycemic agents. Am J Cardiol 2010; 105: 960-966.

14. Patti G, Proscia C, Di Sciascio G. Antiplatelet therapy in patients with diabetes mellitus and acute coronary syndrome. Circ J 2013; 78: $33-41$.

\section{Supplementary Files}

Supplementary File 1

List of Institutions That Participated in or Helped Implement the PRASFIT-ACS Study

Table S1. Treatment-emergency AEs in Japanese patients with acute coronary syndrome undergoing percutaneous coronary intervention

Please find supplementary file(s);

http://dx.doi.org/10.1253/circj.CJ-13-1482 